\title{
EPISTEMOLOGÍA, EDUCACIÓN Y TECNOLOGÍA EDUCATIVA
}

\author{
Flora Eugenia Salas Madriz
}

Recibido 18-VI-2001

Resumen: El artículo hace referencia al trasfondo espitemológico de la Educación y de la Tecnología Educativa, a fin de comprender cuáles son sus nexos teórico-explicativos, así como las proyecciones de aplicación de esta última en el proceso enseñanza-aprendizaje. Posteriormente, se hace un breve recorrido por la fundamentación epistemológica de las Ciencias de la Educación, con el propósito de revisar la compleja red de disciplinas y teorías que nutren el campo de estudio de la Educación. Específicamente, el campo de la Pedagogía, encargada del estudio de la situación y los hechos educativos, toma su basamento epistemológico y teórico de los paradigmas dominantes en Psicología a lo largo del siglo XX. De ellos deriva un marco de acción en la Pedagogía, del cual surgen didácticas específicas, en las cuales la Tecnología Educativa desempeña diversas funciones.

Con base en el hilo conductor de la exposición, se puede concluir que la Tecnología Educativa constituye una herramienta para enseñazar que puede ser implementada, dependiendo del paradigma educativo de que se trate; esto es, forma parte de la didáctica. Se propone, así, que la Tecnología Educativa puede ser estudiada en dos niveles: como disciplina y como herramienta didáctica.
Es nuestro interés abordar el trasfondo espitemológico de la Educación y de la Tecnología Educativa, a fin de comprender cuáles son sus nexos teórico-explicativos, así como las proyecciones de aplicación de esta última en el proceso enseñanza-aprendizaje. Antes de iniciar el abordaje filosófico de estas disciplinas, resulta esclarecedor hacer una breve reflexión sobre la importancia del debate epistemológico en general, y en Educación en particular. Para empezar, debemos recordar que el siglo XX fue un crisol de propuestas epistemológicas donde el eje de la discusión giró alrededor del positivismo, para dar como resultado diversas propuestas epistemológicas (estructuralismo, funcionalismo), así como epistemologías críticas (Habermas) y sociológicas (Kuhn). Esta prolífica discusión aún no está terminada y, por ello, resulta necesario allanar el territorio epistemológico en el cual nos desenvolvemos en el campo de la Educación, con el propósito de no caer en contradicciones e inconsistencias en este ámbito, que invaliden nuestras propuestas teóricas, metodológicas y prácticas.

Como consecuencia de esta rica y vasta discusión, una serie de posiciones y paradigmas han surgido a lo largo del siglo pasado, y cada una de ellas tiene su especificidad. Conocer esta diversidad y tener claridad en el campo de la epistemología permite un máximo aprovechamiento del 
acervo teórico desarrollado hasta ahora, así como la orientación para dar carácter de cientificidad al diseño o ajuste de propuestas educativas concretas. En el entendido de ésto, a continuación se ofrece un breve bosquejo de dos de los paradigmas educativos más importantes del siglo $\mathrm{XX}$, el conductista y el constructivista, así como de la Tecnología Educativa como disciplina y como herramienta didáctica.

\section{El positivismo: características y crítica}

Desde el nacimiento de las ciencias sociales en el siglo XIX, a partir del pensamiento de Augusto Comte (1798-1857), se pretendió que los fenómenos sociales fueran abordados a partir de la lógica de investigación de las ciencias naturales, que ya tenían una importante trayectoria e innumerables éxitos cosechados y reconocidos en la Física, la Química, la Astronomía y la Biología, entre otras. Por esta razón, no es arbitrario que disciplinas nuevas en el ámbito de las ciencias sociales como la Psicología, que nace en 1879 , en un laboratorio en Leipzig, donde Wilhelm Wundt (1832-1920), esperaba "entrar en contacto" y dar cuenta experimental de la conducta humana, emergieran dentro de la lógica de investigación de las ciencias naturales. No obstante, el siglo XIX también vió nacer importantes propuestas teóricas no positivistas como el Marxismo y el Psicoanálisis. Curiosamente, esta última teoría entra en la escena científica en 1900, con la publicación de "La interpretación de los sueños", en la que Freud (1981) propone sus hallazgos sobre el inconsciente. Así, el punto de inflexión entre el siglo XIX y el XX, auguraba ya la discusión por venir en torno a la ciencia, sus características y condiciones en el ámbito de las ciencias sociales, a partir de una teoría revolucionaria que apostaba sus fundamentos epistemológicos en fuentes no positivistas.
La posición epistemológica del positivismo es el empirismo, propuesta que postula que el conocimiento se adquiere a través de la experiencia sensible, $y$, por ende, que la realidad es fenoménica y, por ello, es observable, medible y manipulable. Desde esta perspectiva, la realidad existe independientemente de que haya una mente que la piense, y todos los hallazgos realizados a través de la manipulación intencionada de la realidad son verificables. El método que corresponde a esta posición es el método científico, articulado por la experimentación y la lógica hipotético-deductiva.

El carácter hipotético-deductivo del método científico hace de las matemáticas una herramienta indispensable para la generación de conocimiento reconocido como científico. Así, el uso de los lenguajes lógico-formales, matemático y estadístico, permiten dar cuenta de la realidad a nivel teórico, metodológico y práctico (Cfr.: Anguera, Ma. Teresa, 1998), a partir de enunciados científicos válidos, confiables y verificables experimental y lógicamente. Como se verá, esta poderosa metodología de las ciencias naturales, exitosa y eficiente, presentaba un panorama tentador para quienes quisieron generar conocimiento en el ámbito de las ciencias sociales. Sin embargo, conforme estas ciencias fueron avanzando en sus procesos de investigación, se encontró que el "método único de la ciencia”, tenía fuertes limitaciones para explicar el fenómeno social.

Entre las principales limitaciones que se encontró al aplicar el método científico en las ciencias sociales está, precisamente, su fundamentación epistemológica; es decir, el concebir que la realidad fenoménica es observable, manipulable, verificable y predecible, y que sólo es posible acceder al conocimiento a través de la experiencia sensible. Esto, por cuanto el fenómeno social sólo aparece como tal parcialmente. Cuando sucede, por ejemplo, una huelga de trabajadores, es un hecho que podemos "observarla"; vemos a las personas caminando 
por las calles y ondeando sus pancartas para hacer valer sus peticiones ante la instancia que corresponda, en un momento y lugar específicos. Sin embargo, ahí, en las calles, entre los trabajadores y en su discurso, sólo se pone en evidencia un aspecto de su práctica social, de la cual, sin más información, no podemos emitir un criterio científico. La observación del fenómeno es parcial por cuanto las raíces del descontento se encuentran en diferencias de intereses entre sectores sociales, que se concretan en prácticas concretas. Para abordar este tipo de fenómeno, es necesario profundizar en las causas del problema que, normalmente, implica complejas redes de intereses, actores sociales y políticas, en un contexto histórico particular, independientemente de que el conflicto ocurra en el sector público o en el privado. Por esta razón, dar cuenta "científica" de estos fenómenos excede de varias maneras al método científico y al abordaje epistemológico empirista.

La matemática y la estadística resultan también insuficientes para abordar el fenómeno social o el psicológico, ya que es difícil encontrar mecanismos formalizados o formalizables, para dar cuenta de cosas como "fuerzas sociales", "intereses", "demandas" o "aspiraciones". Todas estas categorías suponen elementos cualitativos que serían dejados por fuera en un abordaje positivista. De hecho, si se ponen estas cosas en perspectiva, los fenómenos sociales y psicológicos quedarían por fuera del conocimiento científico y sería inútil, e incluso ridículo, tratar de dar cuenta de ellos por esta vía.

Otro punto en contra del positivismo estriba en su exclusión de la subjetividad del investigador ya que, en lo que respecta a fenómenos sociales o psicológicos, debido a que en ellos no todo "aparece", es necesario "reconstruir" e "interpretar" eventos, a fin de establecer nexos y criterios de análisis para "comprender" la historia o la biografía del sujeto. Esto nos permite entrar en el problema epistemológico de la "explicación" y la "comprensión". La explicación sólo nos ofrece un cuadro lineal y no necesariamente conectado de los eventos, mientras que la comprensión, además de explicar, ofrece al sujeto de conocimiento la posibilidad de encontrar el "sentido"; es decir, de evaluar el fenómeno al interior de un contexto histórico en el que se rompe el esquema de cadena causal lineal, para entrar en el de complejidad y multicausalidad (Cfr.: Delgado, 1998). Por esta razón, el sujeto participa de manera activa en la reconstrucción e interpretación de los fenómenos, motivo por el cual no es posible excluir su subjetividad, que implica su biografía y su historia y, por ende, su propio contexto cultural.

Lo anterior nos permite argumentar que la realidad social no es replicable, en el sentido en que es replicable, por ejemplo, llevar agua a su punto de ebullición. Puede haber muchas huelgas de trabajadores en el futuro y la única certeza que tenemos es que no serán iguales a las del pasado; por el contrario, siempre que sometamos el agua a una temperatura de cien grados centígrados, obtendremos, invariablemente, su punto de ebullición. La ciencia social siempre es producida al interior de un contexto sociohistórico y cultural y, por ende, siempre da cuenta de fenómenos que son irrepetibles.

Cómo se analice un fenómeno social implica también una posición ética y estética; es decir, una noción particular de "deber ser" reconocida colectivamente, en un momento histórico dado (Cfr.: Singer, 2000). Por tal razón, la ciencia social no puede obviar las dimensiones ética y estética, así como las implicaciones de sus hallazgos, teorías y prácticas. Evidentemente, ésto atañe también a las ciencias naturales ya que ellas son producidas, a su vez, al interior de un contexto, por seres humanos y para ser puestas en escena a nivel social.

Para resumir lo dicho hasta aquí, resulta claro que en ciencias sociales no es posible replicar ni "experimentar", en el 
sentido en que se hace en ciencias naturales. La generalización es exclusiva de una posición inductiva, en donde a través de la observación de un número significativo de eventos se puede asumir que "todos" los eventos de ese tipo serán siempre de esa forma, sin diferencias significativas (Cfr.: Mardones, 1991). La matematización de hechos de naturaleza cualitativa siempre es reduccionista o, eventualmente, imposible. Por tal motivo, la estadística aplicada a las ciencias sociales sólo permite tener ideas generales sobre fenómenos que, para ser comprendidos, requieren de un abordaje complementario basado en métodos y técnicas de investigación cualitativos.

Lo anterior no significa que la estadística o la matematización de ciertos aspectos de la realidad social sea estéril. Lo único que esperamos decir aquí, es que estas metodologías pueden resultar insuficientes para explicar y comprender fenómenos sociales. Dependiendo de cuál sea la intención y los objetivos del investigador o investigadora, así serán los recursos teórico-metodológicos que tiene a $\mathrm{su}$ disposición. Por tal motivo, no hay teorías o métodos mejores o peores, sólo hay limitaciones en ellos que deben ser conocidas y asumidas por quienes investigan, a fin de poder alcanzar las metas propuestas. He aquí la importancia de tener claridad epistemológica, ya que en ella queda comprometida la consistencia científica de toda investigación, independientemente de si se parte de un enfoque positivista o naturalista. Para ejemplificar lo dicho hasta ahora, se utilizará a las Ciencias de la Educación mismas, como veremos a continuación.

\section{Las ciencias de la educación: su fundamentación epistemológica y teórica}

Cuando se dice "ciencias de la educación”, se supone que no hay una disciplina tal que de cuenta por ella misma del fenómeno educativo, sino que se involucran una serie de disciplinas que, en conjunto, pretenden explicar y comprender la compleja trama de elementos que entran en juego en el proceso enseñanza-aprendizaje, en el que confluyen aspectos sociales, psicológicos y comunicacionales. Por esta razón, resulta necesario hacer una clasificación de estas disciplinas, según su objeto de estudio, como se muestra en el siguiente esquema:

\section{Esquema No. 1}

Ciencias de la Educación, según su objeto de estudio

\begin{tabular}{|c|c|c|}
\hline $\begin{array}{l}\text { Estudio de las condi- } \\
\text { ciones generales y lo- } \\
\text { cales de la educación }\end{array}$ & $\longrightarrow$ & $\begin{array}{l}\text { Sociología y } \\
\text { economía de la } \\
\text { educación }\end{array}$ \\
\hline $\begin{array}{l}\text { Estudio de la situa- } \\
\text { ción educativa y los } \\
\text { hechos educativos }\end{array}$ & - & $\begin{array}{l}\text { Psicología de la } \\
\text { educación y } \\
\text { pedagogía }\end{array}$ \\
\hline $\begin{array}{l}\text { Estudio de la evolu- } \\
\text { ción y reflexión de la } \\
\text { educación }\end{array}$ & $\rightarrow$ & $\begin{array}{l}\text { Historia y } \\
\text { filosofía de la } \\
\text { educación }\end{array}$ \\
\hline $\begin{array}{l}\text { Estudio de la orga- } \\
\text { nización educativa }\end{array}$ & 7 & $\begin{array}{l}\text { Administración } \\
\text { educativa }\end{array}$ \\
\hline
\end{tabular}

Adaptado de: Hernández, G. Fundamentos del desarrollo de la Tecnología Educativa (Bases sociopsicopedagógicas). México: OEA-ILCE, 1996. (Modificado por Flora Salas Madriz).

Nótese como las distintas ciencias que abordan el fenómeno educativo, poseen objetos de estudio propios, disciplinas afines, que les aportan elementos teóricos y metodológicos y, por ende, sus fundamentos epistemológicos. Por esta razón, dado que el objetivo de este trabajo es establecer los nexos entre Educación y Tecnología Educativa, nos limitaremos al estudio de la epistemología de la Psicología 
de la Educación y la Pedagogía, que tienen a su cargo el estudio de la situación educativa y los hechos educativos, donde incide directamente la Tecnología Educativa, como veremos más adelante.

Existe una estrecha relación entre las Ciencias de la Educación y las disciplinas de las cuales derivan sus fundamentos generales. Así, la Psicología y sus diversos pardigmas le ofrecen a la Educación un amplio panorama de posibilidades desde las cuales abordar el fenómeno que estudian. Por esta razón, de cada paradigma de la Psicología ha derivado una pedagogía y, entre las más importantes, podemos citar las pedagogías conductista, la cognitiva, la constructivista y la social. Cada una de estas pedagogías toma de base la fundamentación epistemológica de la Psicología que le corresponde. Para ejemplificar lo anterior, partiendo de la noción de paradigma de Kuhn (Cfr.: Mardones, 1991), a continuación se exponen los paradigmas conductista, constructivista y cognitivo, así como las pedagogías a las que dan origen.

\section{El paradigma conductista}

Para J. B. Watson, fundador de la psicología conductista a principios del siglo XX, la Psicología debía ocuparse del estudio de los procesos observables de la conducta humana (Hernández, 1998). Con esta definición del objeto de estudio de la Psicología, la colocaba cómodamente dentro de la corriente positivista y excluía de su interés investigativo todo aquello que no fuera observable, medible y verificable. Así, nociones como la "conciencia” y fenómenos como los sueños, quedaban excluídos del análisis conductista. Por esta razón, sus fundamentos epistemológicos se encontraban en el empirismo, el ambientalismo, el asociacionismo y el anticonstructivismo. Empirismo, ya que la conducta era accesible a través de la experiencia sensible; el ambientalismo por cuanto éste proporciona todos los insumos de la conducta; el asociacionismo porque la mente opera a partir de asociación de ideas simples que permiten la generación de ideas complejas; y el anticonstructivismo ya que el sujeto no tiene control sobre los estímulos que recibe del ambiente. Además, el propósito del conductismo no es otro que moldear la conducta a través de la manipulación intencionada del ambiente.

Esta breve exposición de los fundamentos epistemológicos del conductismo, nos permite elaborar ahora un esquema explicativo de la Pedagogía conductista.

Esquema No. 2

La Pedagogía conductista

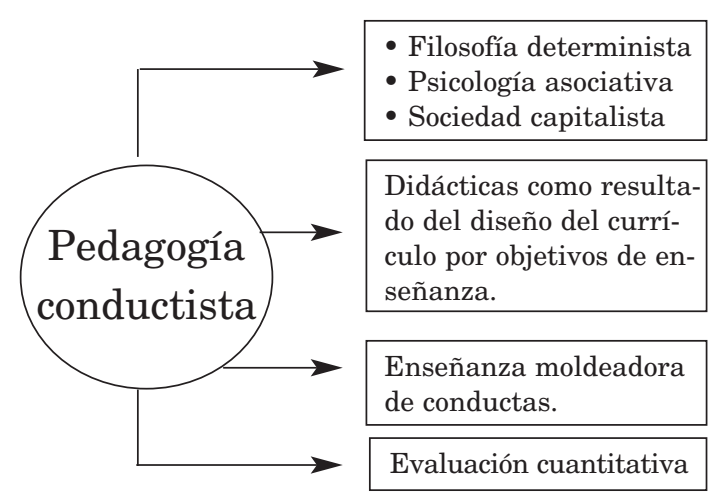

Adaptado de: Flores, R. Evaluación pedagógica y cognición. Colombia: MacGraw Hill, 2000.

Nótese como la pedagogía conductista toma sus fundamentos de la filosofía determinista y la epistemología de la Psicología Asociativa o conductista. Además, el contexto en el que esta forma de ver y pensar el mundo coincide con el proyecto social del capitalismo, en donde el sujeto adquiere su valor social de acuerdo a su capacidad de adaptarse de manera estable.

Consecuente con los fundamentos filosóficos, la Pedagogía conductista elabora didácticas que permiten el logro de objetivos determinados cuyo fin es moldear la conducta en función de los 
intereses sociales. Además, como su método de enseñanza se basa en objetivos, éstos pueden ser evaluados en forma cuantitativa y de manera progresiva, yendo de lo más simple hasta lo más complejo. Resultados numéricos permitirán la promoción o reprobación de los estudiantes, con base en notas basada en medias estadísticamente validadas.

Entre las ventajas que esta pedagogía ofrece están el que se parte de supuestos generales en donde se espera que:

1. Todos los individuos aprendan igual a través de metodologías iguales.

2. El material didáctico que se diseña debe funcionar en todos los sectores y regiones de igual modo.

3. Es posible establecer estadísticamente estándares de excelencia y las escalas son aplicables bajo el criterio de que toda población se comporta en forma "normal".

4. Las excepciones pueden ser tratadas de manera específica (tendencias sub y supranormales).

Esta pedagogía tiene, como ventaja adicional, el abaratamiento de los costes educativos por alummno(a), ya que la capacitación docente, así como el material didáctico y las condiciones materiales del aprendizaje pueden ser estandarizados. Además, permite el abordaje masivo de las necesidades de educación.

\section{El paradigma constructivista}

El paradigma constructivista fue desarrollado por Jean Piaget (1896-1980). A su trabajo también se le ha denominado epistemología genética, ya que su interés investigativo fue la evolución mental en el niño, para determinar cómo se construye el conocimiento humano, en función de los procesos de naturaleza fisiológica y psicológica involucrados en el tránsito de un estadio de menor conocimiento a otro de mayor conocimiento. Por esta razón, la problemática a la que se aboca el constructivismo es la construcción del conocimiento. Sus fundamentos epistemológicos son el constructivismo, el interaccionismo y el relativismo (Hernández, 2000). Constructivismo porque parte del supuesto de que el sujeto es activo ante el entorno, tomando de éste aquellos elementos que le resultan significativos. Interaccionismo, por la misma razón; esto es, ya que el sujeto interactúa con su entorno para poder resolver los problemas que se le presentan, y relativismo ya que, dependiendo del entorno y de las necesidades del sujeto, los procesos de conocimiento se desarrollan, generando resultados específicos, para situaciones específicas.

Entre los principales supuestos teóricos de este paradigma tenemos la teoría de las etapas del desarrollo, en donde se logra establecer que todo ser humano se encuentra determinado por invariantes funcionales, genéticamente constituidas, así como por el ambiente -natural y social-, en el que el sujeto se ve movilizado del desequilibrio al equilibrio a lo largo de su vida.

Piaget desarrolla un método específico que es el psicogenético, en el cual se estipulan las metodologías y técnicas de investigación necesarias para evaluar las distintas etapas del desarrollo del sujeto, que le permiten acceder al lenguaje y al conocimiento.

Como consecuencia de esta Psicología, nace la pedagogía constructivista, como se muestra en el esquema siguiente: 
Esquema No. 3

La Pedagogía constructivista

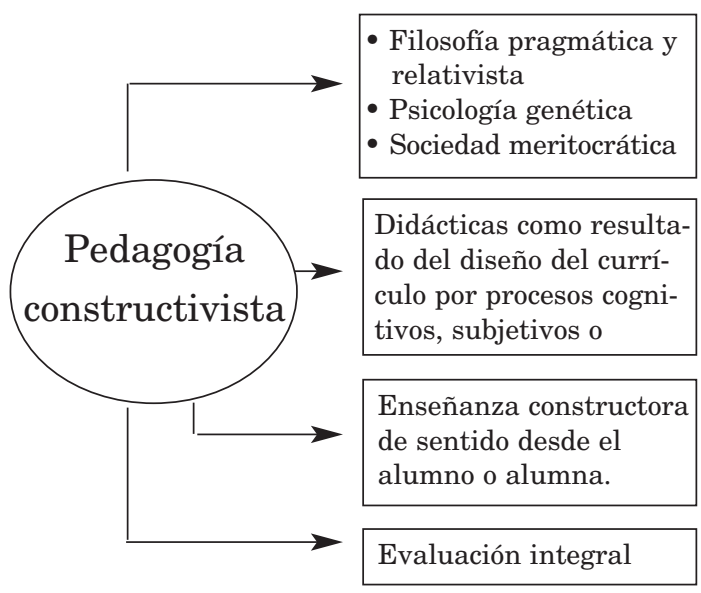

Adaptado de: Flores, R. Evaluación pedagógica y cognición. Colombia: MacGraw Hill, 2000.

Nótese como la pedagogía constructivista toma sus fundamentos de la filosofía pragmático-relativista y la epistemología de la Psicología genética. Además, el contexto en el que esta forma de ver y pensar el mundo coincide con el proyecto social meritocrático, en el que los individuos alcanzan su valor social en función de su desempeño y de su capacidad de respuesta al cambio.

Consecuente con los fundamentos filosóficos, la Pedagogía constructivista, elabora didácticas que permiten el logro de procesos cognitivos en donde lo subjetivo -construcción de significados- y la investigación -conocimiento desarrollado por el propio sujeto-, son fundamentales a nivel curricular. La evaluación, al ser integral, debe involucrar aspectos de naturaleza cualitativa, además de los de naturaleza cuantitativa.

Entre las ventajas que esta pedagogía ofrece están el que se parte de supuestos generales en donde se espera que:

1. Los sujetos aprendan determinados por sus invariantes funcionales -genéticos-, y ambientales.
2. Los sujetos aprendan en forma diferente en contextos diferentes.

3. El sujeto debe hacer significativo el conocimiento para que se produzca auténtico aprendizaje.

4. La investigación es una estrategia clave para aprender.

Como uno de los principales problemas que tiene la implementación de esta pedagogía está el elevado costo por alumno(a), ya que supone grupos relativamente pequeños de estudiantes por docente, así como docentes altamente capacitados y la disponibilidad de recursos didácticos no tradicionales.

Vemos así que para implementar una auténtica pedagogía constructivista se requiere de un abordaje individualizado, contextualizado y regionalizado de la educación.

\section{La tecnología educativa}

La tecnología educativa tiene su origen en la década de los años cincuenta, en los Estados Unidos, cuando se estudió el problema de las diferencias individuales en el aprendizaje y se investigó la posibilidad de desarrollar dispositivos de autoenseñanza en el entrenamiento militar. $\mathrm{Pa}$ ra entonces, la Unión Soviética había alcanzado un importante desarrollo a nivel científico en general y, en particular, en lo que respecta a la conquista del espacio. De igual manera, se había convertido en una potencia militar, razón por la cual los Estados Unidos se vieron comprometidos a mejorar de manera significativa la educación, la investigación científica y el área militar, para responder ante la potencia que se presentaba entonces como su rival por excelencia. La Guerra Fría entraba en el escenario mundial y, como respuesta, los Estados Unidos emprendieron acciones orientadas a cerrar la brecha que los separaba de la superpotencia socialista. 
Estados Unidos contaba además en esa época, con el invento de la computadora, desarrollada en 1946, y se propuso utilizar este dispositivo no sólo en el campo militar, sino también en el civil. Precisamente, uno de los usos civiles de la computadora fue la potenciación de la educación, a través de su incorporación como herramienta didáctica. El desarrollo de pensamiento analítico y científico requiere de un alto grado de habilidades lógico-matemáticas y el computador, debido a su estructura conceptual, permitía desarrollar estas habilidades en forma rápida y eficiente. Bien utilizada, esta nueva tecnología podía convertirse en una herramienta de gran poder didáctico.

Como sabemos, la pedagogía predominante en esta época era la conductista, razón por la cual los investigadores del campo se dieron a la tarea de evaluar las aplicaciones de la computadora en la enseñanza. Skinner hizo importantes contribuciones en este campo y desarrolló elementos teórico-metodológicos que hicieron posible diseñar dispositivos de aprendizaje utilizando esta nueva tecnología.

Además del desarrollo teórico en el campo de la pedagogía conductista, es importante señalar que en la década de los años ciencuenta, fueron desarrolladas nuevas tecnologías audiovisuales, derivadas de la industria del cine, de la televisión y, como innovación, el videotape, desarrollado por Ampex a finales de esta década, surgiendo como el primer sistema de registro magnético de la señal de vídeo. De esta manera, la tecnología educativa y el aprendizaje han quedado enlazados a partir de la propuesta de la instrucción programada, desarrollada desde el enfoque conductista (Cfr.: Bartolomé, 2001).

Vemos como confluyeron una serie de factores de naturaleza tecnológica, educativa, política y social, que propiciaron la aparición de la tecnología educativa, abriendo un nuevo campo de estudio a nivel pedagógico, así como nuevas posibilidades a nivel comercial, social y cultural. Los dispositivos desarrollados para la difusión masiva de mensajes, capaces de transmitir imagen, movimiento y sonido, revolucionaron nuestro concepto de aprendizaje, al revolucionar nuestro concepto del mundo, de la realidad y de nosotros mismos.

Según la UNESCO (Citado por Bartolomé, 2001), la Tecnología Educativa "es un modo sistemático de concebir, aplicar y evaluar el conjunto de los procesos de enseñanza-aprendizaje". De tal manera, implica un ámbito básico de estudio que gira en torno de ella y de la cual derivan sus fundamentos epistemológicos, teóricos y metodológicos propios. Con esto queremos decir que la Tecnología Educativa constituye un campo nuevo de estudio, con su problemática propia, así como con sus proyecciones de aplicación en el campo educativo, como puede observarse en el siguiente esquema (Cfr.: Bartolomé, 2001).

Esquema No. 4

Ámbito básico de la tecnología educativa

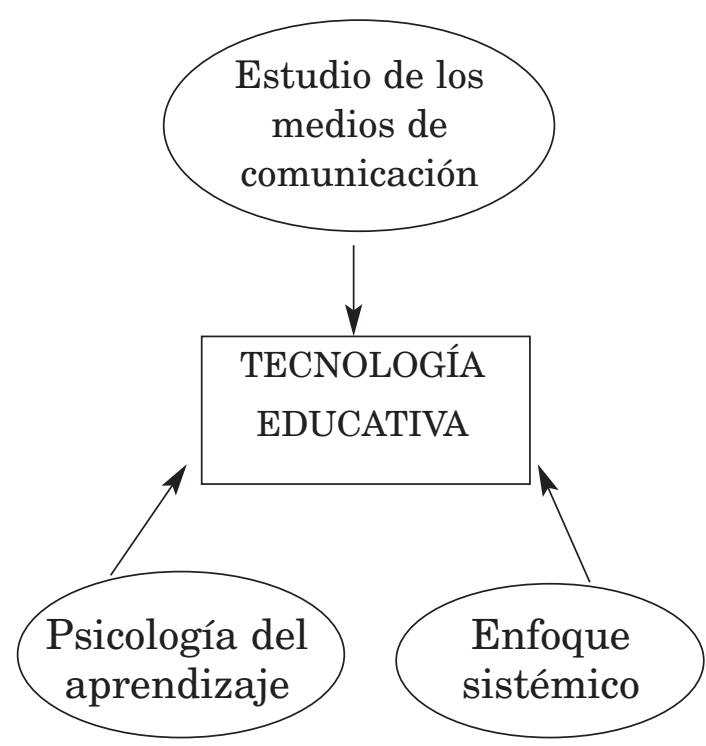


Como puede observarse en el esquema anterior, la Tecnología Educativa abre una serie de problemáticas completamente nuevas, que involucran lo específicamente tecnológico; esto es, lo que tiene que ver con los medios de comunicación, en tanto dispositivos, así como sus posibilidades comunicacionales. La Psicología del Aprendizaje, ya que impacta de manera diversa a las distintas pedagogías y a las didácticas que de ellas derivan. Finalmente, el enfoque sistémico, ya que éste permite tener una perspectiva más amplia de la Tecnología Educativa, llevándola a niveles sociales sin precedentes, como son los canales de televisión educativos, de los cuales son un buen ejemplo el Canal Discovery y el History Chanel -E.U.A.-, Televisión Educativa española, o el canal de la National Geographic -Inglaterra-.

Vemos, entonces, que existe una relación entre Pedagogía y Tecnología Educativa, ya que el objeto de estudio de la Pedagogía “... es el estudio y diseño de experiencias culturales que conduzcan al progreso individual en su formación humana", [y la didáctica] “... es un área de estudio de la pedagogía, el más instrumental y operativo, pues se refiere a las metodologías de enseñanza, al conjunto de métodos y técnicas que permiten enseñar con eficacia" (Flores, 2000: XIX-XXIII). La Tecnología Educativa constituye una herramienta para enseñar que puede ser implementada, dependiendo del paradigma educativo de que se trate; esto es, forma parte de la didáctica.

Para resumir, hemos podido ver como la Tecnología Educativa puede ser estudiada en dos niveles: como disciplina y como herramienta didáctica. En este último sentido, se deben considerar los siguientes aspectos:

1. La didáctica no se entiende ni se aplica como un conjunto de técnicas comunicativas aisladas de los principios y de la red conceptual que caracteriza a cada teoría pedagógica.
2. Cada teoría pedagógica y cada modelo pedagógico, propone una didáctica diferente.

3. Las directrices generales de la didáctica necesitan acoplarse y asimilarse a las condiciones de cada paradigma educativo específico.

La Tecnología Educativa en sí misma no puede garantizar el convertirse en una herramienta eficiente en el ámbito educativo, sino que ello va a depender del paradigma educativo y de la pedagogía que se elijan, tanto como de la manera en que sea implementada en el ámbito didáctico. $\mathrm{Pa}$ ra aplicar la Tecnología Educativa, es necesario considerar aspectos como la política educativa vigente, la Psicología educativa coherente con tal política, la pedagogía que le corresponda y, con ello, la didáctica derivada en la que se incorporen dispositivos y herramientas desarrollados a nivel tecnológico para su aplicación en educación.

En esta época la Educación cuenta con un amplio espectro de posibilidades pedagógicas, debido al desarrollo de las propuestas paradigmáticas en Psicología. La claridad epistemológica es condición necesaria, aunque no suficiente, para una adecuada utilización de la Tecnología Educativa como herramienta didáctica. Como ejemplo de lo anterior, están las nuevas propuestas psicogenética y sociocultural y las cognitivas, que al compartir la postura epistemológica constructivista, rompen de manera radical con el paradigma tradicional de corte conductista. Sin embargo, en el caso particular de Costa Rica, también es cierto que estas nuevas corrientes pedagógicas han puesto en evidencia las limitaciones para su implementación, debido a la falta de claridad epistemológica y teórica al interior del sistema educativo mismo, donde aún se encuentran presentes en los programas, prácticas didácticas y en la evaluación, posiciones en las que subyace un claro corte epistemológico conductista. 
Finalmente, resulta importante destacar que la Tecnología Educativa refleja el nivel más operacional de una práctica pedagógica concreta y, por ende, de un paradigma educativo que, a su vez, responde y es coherente con la política educativa propuesta a nivel global. Quizá esto nos permita visualizar que los verdaderos problemas en educación deben ser atacados a nivel político primero, y paradigmático después. Sólo así se puede esperar que la incorporación de tecnologías educativas produzca efectos significativos en cuanto a calidad educativa se refiere en el nivel didáctico. Se debe desmitificar a la tecnología educativa, ya que, como se expuso anteriormente, no puede hacer "milagros", ya que su impacto sólo puede ser consecuencia de la estrategia didáctica en la que se le incorpore. De ello depende el grado de éxito potencial de las tecnologías educativas como herramienta didáctica.

\section{Referencias bibliográficas}

Anguera, Ma. Teresa, et al. Métodos de investigación en Psicología. Madrid: Editorial Síntesis, 1998.

Delgado, Juan Manuel y Juan Gutiérrez. Métodos y técnicas cualitativas de investigación en ciencias sociales. Madrid: Editorial Síntesis, 1998.

Flores, Rafael. Evaluación pedagógica y cognición. Colombia: MacGraw Hill, 2000.

Freud, Sigmund. Obras Completas. Madrid: Biblioteca Nueva, 1981.

Hernández, Gerardo. Fundamentos del desarrollo de la tecnología Educativa (Bases sociopsicopedagógicas). México: OEA-ILCE, 1996.

Hernández, Gerardo. Paradigmas en psicología de la educación. México: Paidós, 1998.

Mardones, J.M. Filosofía de las ciencias humanas y sociales. Barcelona: Anthropos, 1991.

Singer, Peter. A companion to Ethics. Great Britain: MPG Books Ltd., 2000.

Bartolomé, Antonio. "Concepción de la tecnología educativa a finales de los ochenta". Antonio.Bartolomé@doe.d5.ub.es [Consulta agosto, 2001]

Flora Salas Madriz Profesora de la Escuela de Administración Educativa de la Universidad de Costa Rica 